\title{
Aerobic Bacterial Profile of Wound Infections and Its Sensitivity Pattern at Tertiary Care Hospital
}

\author{
M. H. Bhalchandra*, S. D. Naik and Pramod Kumar Verma
}

Department of Microbiology, MGM's Medical College and Hospital, Aurangabad, India

*Corresponding author

\begin{tabular}{|l|}
\hline K e y w or d s \\
Wound infection, \\
Bacterial agents, \\
Antibiotic \\
sensitivity
\end{tabular}

A B S T R A C T

A wound is a breach in the skin and the exposure of subcutaneous tissue following loss of skin integrity. Wound infection occurs as a complication of surgery, trauma or disease that may interrupt a mucosal or skin surface. Wound infections are one of the most common hospital acquired infections. They are an important cause of morbidity and mortality. The present study was undertaken to determine the aerobic bacterial agents responsible for different wound infections, its sensitivity pattern and to find out the most common bacterial agent in pathological, trauma and post-operative wound infections. All pus or wound swabs from various wound infections received in the department of Microbiology for culture and sensitivity during January 2012 to December 2012 from IPD and OPD patients attending tertiary care hospital were cultured on $5 \%$ sheep blood agar and MacConkey agar. The bacterial isolates were indentified by standard bacteriological techniques. Isolated organisms were further tested for antibiotic sensitivity, MSRA and ESBL productions. Out of 303 samples, $202(66.66 \%)$ were culture positive in which 140 $(69.31 \%)$ samples were monomicrobil and $62(30.69 \%)$ samples were polymicrobial. Thus total 271 isolates were obtained from 202 culture positive samples. Of the total 271 isolates, $160(59.04 \%)$ were gram negative ans $111(40.96 \%)$ were gram positive organism. The most common isolates was S.aureus $(37.63 \%)$ followed by Pseudomonas spp. $(20.33 \%)$ and E.Coli $(19.56 \%)$, In pathological and post-operative wound infections S.aureus was the most common i.e.44.62\% and $34.09 \%$ respectively. Whereas pseudomonas species was most common in trauma wound infections. Isolated strains of $S$. aureus were $78 \%$ sensitive to Amikacin and $73 \%$ sensitive to Linezoid. E.coli, Klebsiella spp and proteus spp were $81 \%, 57 \%$ and $91 \%$ sensitive to Amikacin respectively. Pseudomonas spp were $96 \%$ sensitive to imipenem and $56 \%$ sensitive to Amikacin. Isolated most of the strains of gram positive and gram negative organisms were sensitive to Amikacin, whereas there was no single common antibiotic to which all isolated gram positive and gram negative bacteria were $100 \%$ sensitive. We found $67.65 \%$ MRSA and $38.12 \%$ ESBL producers in this study.

\section{Introduction}

Skin, the largest organ in human body play a crucial role in the sustense of life through the regulation of water and electrolyte balance, thermoregulation and by acting a barrier to external noxious agents including microorganisms, however when the epithelial 
integrity of the skin disrupted a wound result. (Zafar et al., 2007) A wound is a breach in the skin and exposure of subcutaneous tissue following loss of skin integrity which provides a moist, warm and nutritive environment that is conductive to microbial colonization and proliferation. (Bowler et al., 2001)

Wound infection occurs as a complication of surgery, trauma or disease that may interrupt a mucosal or skin surface. (Njoku-Obi and Ojiegbe, 1989) Development of wound infection depends on the interplay of many factors. The breaking of the host protective layer, the skin, and thus disturbing the protective function of the layer will induce many cell types into the wound to initiate host response. (Collier, 2003) Infection of wound is the successful invasion and proliferation by one or more species of microorganisms anywhere within the body sterile tissue, sometimes resulting in pus formation. (Mordi and Momoh, 2009)

Wound can be classified as accidental, pathological, or post-operative. Irrespective of the wound nature, infection is the attachment of microorganisms to host cells and their proliferation, colonization ultimately leading to host tissue damage. (Collier, 2003). Wound infection can be caused by different group of microorganisms like bacteria, fungi and protozoa. (Zafar, 2007) Bacteriological studies have also shown that wound infection is universal and that the types of bacteria very with geographical location, bacteria resident on the skin, clothing at the site of the wound, time between wound and examination. (Akinjogunla et al., 2009) The infections are caused by both Gram positive and Gram negative bacteria especially by Staphylococcus aureus (S.aureus), Escherichia coli (E.coli) and Pseudomonas aeruginosa (p.aeruginosa) (Bowler and Duerden, 2001).The common Gram positive organisms are the B-hemolytic Streptococci, Streptococcus pyogenes (S. pygenes) and S.aureus. The facultative anaerobes include Enterbacter spp, E.coli, Klebsiella spp, Proteus spp and aerobic Gram negative rods are P.aeruginosa. The fungal organisms are Candida spp and moulds (Aspergillus spp.) (Sani et al., 2012)

Wound infections are one of the most common hospital acquired infections. They are an important cause of morbidity and account for $70-80 \%$ mortality. (Gottarup et al., 2005) Development of such infection represent delayed healing, cause anxiety and discomfort for patient, longer hospital stays and add to the cost of health care services significantly. (Mohanty et al., 2004) The wide spread use of antibiotics together with the length of time over which they have become available have lead to major problem of resistant organisms contributing to high morbidity and mortality. Antimicrobial resistance can increase complications along with increase in cost related to procedure and treatment. (Taiwo et al., 2002) Infection caused by Methicillin-resistant S.aureus (MRSA) and multidrug resistant organisms like extended spectrum beta-lactamase (ESBL) producer pose a major challenge in the treatment of wound infection. So, appropriate drug selected by antibiotic sensitivity testing have great importance. (Kaur et al., 2008)

This study was therefore aimed to determine the bacteriological profile of pathological, traumatic and post-operative wound infections and its sensitivity patterns, which is helpful in the management of these wound infections and formulating a Rational Antibiotic Policy in this hospital.

The main and objectives of this study includes to find out the aerobic bacterial agent responsible for causing pathological, trauma and post-operative wound infections. 
To find out sensitivity pattern of isolated bacterial agents.

To find out commonest bacterial agents responsible for above wounds.

\section{Materials and Methods}

The prospective observational study was conducted after the permission from ethical committee.

An analysis was conducted on 303pus or wound swabs from all types of wound like burns, bed sores, cellulitis, ulcers, abscesses, osteomyelitis, trauma wounds, and postoperative sepsis received in the Department of Microbiology for culture and sensitivity during January 2012 to December 2012 from IPD and OPD patients attending tertiary care hospital. Isolated organisms were tested for antibiotic sensitivity pattern, MRSA and ESBL production.

\section{Inclusion criteria}

Pus and wound swabs from all age group and birth sexes received in the department of Microbiology for aerobic bacterial culture and sensitivity were included in this study.

\section{Exclusion criteria}

Sample for acid fast bacterial culture were excluded from this study.

Two wound swabs (one for Gram stain and another for culture) or pus from different types of wound were processed. Gram staining was done from one swab and findings were recorded. Another swab was used for culture on 5\% sheep blood agar and MacConkey agar. Inoculated plates were incubated at $37^{\circ} \mathrm{C}$ After overnight incubation growth if any was identified by standard bacteriological technique including colony morphology, Gram staining from colony and biochemical properties. (Church et al., 2006; Mackie and McCartney, 1989)

\section{Antibiotic sensitivity testing}

The antibiotic sensitivity of isolated strain was carried out by using Kirby Bauer disc diffusion method on Muller Hinton agar by using commercially available antibiotic discs (Hi Media). (Kirby et al., 1966). The following antibiotics were used for Gram positive and Gram negative strains in the present study.

\section{For gram positive organisms}

Clindamycin $2 \mu \mathrm{g}, \quad$ Cefoxitin $\quad 30 \mu \mathrm{g}$,

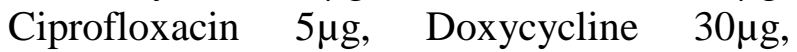
Erythromycin $15 \mu \mathrm{g}, \quad$ Linezoid $30 \mu \mathrm{g}$, Penicillin10 units/disc, Amikacin 30 $\mu \mathrm{g}$, Levofloxacin $5 \mu \mathrm{g}$, Co-trimoxazole $25 \mu \mathrm{g}$.

\section{For Gram negative bacteria}

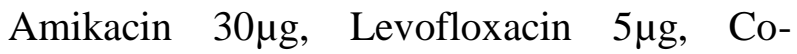
trimoxazole $25 \mu \mathrm{g}$, Amoxycillin- clavulanic

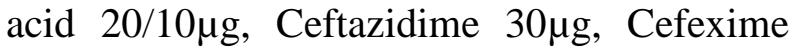
$30 \mu \mathrm{g}$, Cefoperazone $75 \mu \mathrm{g}$, Ceftriaxone $30 \mu \mathrm{g}$, Cefuroxime $30 \mu \mathrm{g}, \quad$ Ofloxacin $5 \mu \mathrm{g}$, Piperacillin-Tazobactum 100/10 $\mu$ g, Imipenem $10 \mu \mathrm{g}$.

The known ATCC standard strain of E.coli (25922), S.aureus (25923) and P. aeuroginosa (27853) were used as quality control throughout the study for culture and antimicrobial testing.

\section{Detection of MRSA: (Clinical and Laboratory Standards Institute, 2012)}

The isolated $S$. aureus strains were tested for MRSA by using cefoxitin disc along with the antibiotic sensitivity testing of different antibiotics used in this study. In case of cefoxitin disc if the Zone of Inhibition was less than or equal to $21 \mathrm{~mm}$ was considered as 
MRSA while more than or equal to $22 \mathrm{~mm}$ zone of inhibition was considered as MSSA(CLSI2012). Quality control was mentioned by using ATCC MRSA (43300) strain.

Detection of ESBL producing strains: (Clinical and Laboratory Standards Institute, 2012)

Gram negative isolates which showed resistance to ceftazidime were tested for ESBL production by ESBL phenotypic confirmatory test. (CLSI, 2012).

Standard inoculum were prepared from 3-4 colonies of identified Gram negative isolates on peptone water in a tube and incubated at $37^{\circ}$ for 4 hours.

After incubation turbidity of inoculums was compared and adjusted to 0.5 McFerlands. Inoculum was inoculated on Muller Hinton agar by using sterile cotton swaband allowed to dry surface of agar for 3-5 minutes.

Then ceftazidime $(30 \mu \mathrm{g})$ and ceftazidime clavulanic acid $(30 / 10 \mu \mathrm{g})$ disc were placed $15 \mathrm{~mm}$ apart on inoculated Muller Hinton agar by using sterile forceps. Similarly cefotaxime $(30 \mu \mathrm{g})$ and cefotaxime-clavulanic acid $(30 / 10 \mu \mathrm{g})$ discs were placed $15 \mathrm{~mm}$ apart. The plates were incubated at $37^{\circ} \mathrm{C}$ for $16-18$ hours.

After incubation zone of inhibition around all these four discs was measured using ruler. A $5 \mathrm{~mm}$ or more increase in zone of inhibition around ceftazidime-clavulanic acid than ceftazidime alone or cefotaxime-clavulanic acid than cefotaxime alone was considered as ESBL producer. ATCC ESBL producer Klebsiella pneumoniae (700603) was used as quality control. (CLSI2012).

\section{Results and Discussion}

A total 303 pus or wound swab samples received in the department of microbiology were subjected for culture and bacterial isolates were identified by standard bacteriological techniques. Isolated organisms were further tested for antibiotic sensitivity, MRSA and ESBL production. Out of 303 samples, 101 were culture negative 202 were culture positive; in which 140(69.31\%) samples were monomicrobial and 62(30.69\%) were polymicrobial. Thus total 271 isolates were obtained from 202 culture positive samples; and following observations were made:

In the present study culture positivity was $66.66 \%$. Out of 202 culture positive samples, $140(69.31 \%)$ samples were monomicrobial and $62(30.69 \%)$ were polymicrobial. Thus total 271 organisms were obtained.

Out of 271 bacterial isolates, 160(59.04\%) isolates were Gram negative and 111(40.96\%) isolates were Gram positive.

Among total the most common isolate was $S$. aureus 102(37.63\%) followed by pseudomonas spp 55 (20.33\%), E.coli 53(19.56\%), Klebsiella spp 37(13.65\%), Proteus spp 11(4.05\%), Streptococcus spp 8(2.95\%), Citobacter, Enterobacter spp 2 each $(0.74 \%)$ and Enterococci spp one (0.34\%).

The percentage of culture positivity among pathological and post-operative wound infection was $67.39 \%$ and $67.33 \%$ respectively. In trauma wound infections it was $57.14 \%$.

In pathological wound infections $100 \%$ culture positivity was seen in bed sores and cellulitis. In ulcers it was $69.86 \%$, burns $69.23 \%$, osteomyelitis $66.66 \%$, abscesses $64.77 \%$ and in others $50.00 \%$.

In the present study, out of total 121 isolates from pathological wound infections, S.aureus was the most common i.e. 54(44.62\%) 
followed by E.Coli 24 (19.83\%), Pseudomonas spp 18 (14.88\%), Klebsiella spp 14 (11.57\%), Streptococcus spp 5 (4.13\%), Proteus spp 4 (3.31\%), Citrobacter spp and Enterococci spp one $(0.83 \%)$ each.

Out of 18 isolates in trauma wound infection, Pseudomonas spp was the most common isolates i.e $6(33.33 \%)$, in trauma/ RTA wound infactions followed by E.Coli 5(27.77\%), S.aureus and Klebsiella spp 3 (16.67\%) each; and Proteus spp one (5.56\%). In this study even a single sample of animal/insect bite wound infection was not received.

Out of 132 isolates from post-operative wound infections, 45 (34.09\%) isolates were S.aureus the most common isolates followed by Pseudomonas spp 31 (23.48\%), E.Coli 24 (18.18\%), Klebsiella spp 20 (15.15\%), Proteus spp 6 (4.54\%). Streoptococcus spp 3 (2.27\%), Enterobacter spp $2(1.52 \%)$ and Citrobacter spp one $(0.76 \%)$.

In the present study, the most common organism in pathological and postoperative wound infection was S.aureus i.e. 54 (44.62\%) and $45(34.09 \%)$ respectively. In trauma wound infection commonest organism was Pseudomonas spp i.e. 6 (33.33\%).

In the present study isolated S.aureus were $78 \%$ sensitive to amikacin, $75 \%$ to linezolid, $70 \%$ to levofloxacin, $68 \%$ to doxycycline and $54 \%$ sensitive to clindamycin.

Pseudomonas spp were $96 \%$ sensitive to imipenem, 56\% sensitive to amikacin and $51 \%$ sensitive to levofloxacin.

E.Coli were $81 \%$ sensitive to amikacin, $42 \%$ sensitive to levofloxacin and $38 \%$ sensitive to piperacillin-tazobactum.

Klebsiella spp were $57 \%$ sensitive to amikacin and $49 \%$ sensitive to levofloxacin. Whereas $100 \%$ resistant to amoxycylin + clavulanic acid, cefexime and cefuroxime.

Proteus spp were sensitive to amikacin, levofloxacin and piperacillin tazobactum, $91 \%, 82 \%$ and $82 \%$ respectively.

Streptococcus spp were $30 \%$ sensitive to amikacin, $57 \%$ and $63 \%$ sensitive to levofloxacin and doxycycline respectively.

Citrobacter spp were $50 \%$ sensitive to amikacin, levofloxacin, piperacillin tazobactum and ofloxacin. Whereas $100 \%$ resistant to cotrimoxazole, amoxycycline clavulanic acid, ceftazidime, cefexime, cefoperazone, ceftrizone, cefuroxime.

Enterobacter sppwere $100 \%$ sensitive to amikacin, levofloxacin, piperacilline tazobactum, ofloxacin and were $100 \%$ resistant to amoxycilline clavulanic acid, ceftazidine, cefexime and cefuroxime.

Enterococci spp were $100 \%$ sensitive to mikacin, levofloxacin, linezolid, doxycycline and clindamycin. Whereas $100 \%$ resistant to ciprofloxacin, erythromycin, penicillin, cotrimoxazole.

Isolated most of the strains of Gram positive (S.aureus, Streptococcus spp and Enterococci spp) and Gram negative (E.Coli, Proteus spp, Enterobacter spp) organisms were sensitive to amikacin.

Whereas there was no single common antibiotic to which all isolated Gram positive and Gram negative bacteria were $100 \%$ sensitive.

Out of 102 S.aureus isolates 69(67.65\%) were MRSA and out of 160 Gram negative isolates, 61(38.12\%) were ESBL producers. 
Table.1 Culture positivity in different sub types of wound infections

\begin{tabular}{l|l|l|l|l|}
$\begin{array}{l}\text { Nature of wound } \\
\text { infections }\end{array}$ & $\begin{array}{l}\text { Total no of } \\
\text { samples }\end{array}$ & $\begin{array}{l}\text { No of samples } \\
\text { showing growth }\end{array}$ & $\begin{array}{l}\text { Sterile samples } \\
\text { sa of culture } \\
\text { positivity }\end{array}$ \\
\hline $\begin{array}{l}\text { A. Pathological } \\
\text { 1. Burn }\end{array}$ & 13 & 9 & 4 & $69.23 \%$ \\
$\begin{array}{l}\text { 2. Bed sore } \\
\text { 3. Cellulitis }\end{array}$ & 2 & 2 & 0 & $100 \%$ \\
4. Ulcer & 23 & 5 & 0 & $100 \%$ \\
\hline $\begin{array}{l}\text { 5. Abscess } \\
\text { 6. Osteomyelitis }\end{array}$ & 38 & 16 & 7 & $69.86 \%$ \\
7. others & 4 & 57 & 31 & $64.77 \%$ \\
\hline $\begin{array}{l}\text { B. Trauma } \\
\text { Trauma/RTA }\end{array}$ & 21 & 2 & 1 & $66.66 \%$ \\
\hline C. Postoperative & 144 & 2 & 2 & $50.00 \%$ \\
\hline Total & 303 & 12 & 9 & $57.14 \%$ \\
\hline
\end{tabular}

Table.2 Organisms isolated from different sub types of pathological wound infection

\begin{tabular}{|l|l|l|l|l|l|l|l|l|}
\hline $\begin{array}{l}\text { Organism } \\
\text { isolated }\end{array}$ & $\begin{array}{l}\text { Toatal no. of } \\
\text { isclates (\%) }\end{array}$ & Burn & Bedsore & $\begin{array}{l}\text { Cellul } \\
\text { ities }\end{array}$ & Ulcer & Abscess & $\begin{array}{l}\text { Osteo } \\
\text { mylitis }\end{array}$ & Other \\
\hline $\begin{array}{l}\text { S.aureus } \\
54(44.62 \%)\end{array}$ & 3 & 1 & 1 & 6 & 40 & 2 & 1 \\
\hline E.Coli & $24(19.83 \%)$ & - & 1 & - & 4 & 19 & - & - \\
\hline $\begin{array}{l}\text { Pseudomonas } \\
\text { spp }\end{array}$ & $18(14.88 \%)$ & 7 & - & 2 & 5 & 4 & - & - \\
\hline $\begin{array}{l}\text { Klebsiella spp } \\
\text { 14(11.57\%) }\end{array}$ & 2 & - & 1 & 7 & 3 & - & 1 \\
\hline $\begin{array}{l}\text { Streptococcus } \\
\text { spp }\end{array}$ & $5(4.13 \%)$ & - & - & 3 & 2 & - & - & - \\
\hline Proteus spp & $4(3.31 \%)$ & - & 1 & - & 1 & 2 & - & - \\
\hline $\begin{array}{l}\text { Citrobacter } \\
\text { spp }\end{array}$ & $1(0.83 \%)$ & - & - & - & - & 1 & - & - \\
\hline $\begin{array}{l}\text { Enterococci } \\
\text { spp }\end{array}$ & $1(0.83 \%)$ & 1 & - & - & - & - & - & - \\
\hline \begin{tabular}{l} 
Total \\
\hline
\end{tabular} & $121(100 \%)$ & 13 & 3 & 7 & 25 & 69 & 2 & 2 \\
\hline
\end{tabular}

Table.3 Organisms isolated from sub types of trauma wound infections

\begin{tabular}{|l|l|l|}
\hline Organisms isolated & Total no. of isolates & Trauma/RTA \\
\hline Pseudomonas spp & $6(33.33 \%)$ & 6 \\
\hline E.Coli & $5(27.77 \%)$ & 5 \\
\hline S.aureus & $3(16.67 \%)$ & 3 \\
\hline Klebsiella spp & $3(16.67 \%)$ & 3 \\
\hline Proteus spp & $1(5.56 \%)$ & 1 \\
\hline Total & $\mathbf{1 8 ( 1 0 0 \% )}$ & $\mathbf{1 8}$ \\
\hline
\end{tabular}


Table.4 Organisms isolated from postoperative wound infection

\begin{tabular}{|l|l|}
\hline Organisms isolated & Total no.of organisms (\%) \\
\hline S.aureus & $45(34.09 \%)$ \\
\hline Pseudomonos spp & $31(23.48 \%)$ \\
\hline E.Coli & $24(18.18 \%)$ \\
\hline Klebsiella spp & $20(15.15 \%)$ \\
\hline Proteus spp & $06(4.54 \%)$ \\
\hline Streptococcus spp & $03(2.27 \%)$ \\
\hline Enterobacter spp & $02(1.52 \%)$ \\
\hline Citrobacter spp & $01(0.76 \%)$ \\
\hline Total & $\mathbf{1 3 2}(\mathbf{1 0 0 \%})$ \\
\hline
\end{tabular}

Table.5 Most common isolates from pathological, trauma and post-operative wound infections

\begin{tabular}{|l|l|l|l|}
\hline Nature of wound & Most common organism & No. of isolates & $\%$ of most common isolates \\
\hline Pathological & S.aureus & $54(\mathrm{n}=121)$ & $44.62 \%$ \\
\hline Trauma & Pseudomonas spp & $06(\mathrm{n}=18)$ & $33.33 \%$ \\
\hline Postoperative & S.aureus & $45(\mathrm{n}=132)$ & $34.09 \%$ \\
\hline
\end{tabular}

Table.6 Drug sensitivity (\%) pattern of isolates from different type of wound infection

\begin{tabular}{|c|c|c|c|c|c|c|c|c|c|c|c|c|c|c|c|c|c|c|c|c|}
\hline $\begin{array}{l}\text { Organism } \\
\text { isolated(n=) }\end{array}$ & & CD & CX & CIP & DO & E & LZ & $\mathbf{P}$ & $\mathbf{A K}$ & LE & $\begin{array}{l}\text { Co } \\
\text { T }\end{array}$ & AMC & CAZ & CFM & CPZ & $\begin{array}{l}\mathrm{CT} \\
\mathrm{R}\end{array}$ & CXM & OF & PIT & IPM \\
\hline $\begin{array}{l}\text { S.aureus } \\
(\mathrm{n}=102)\end{array}$ & $\mathrm{R}$ & $\begin{array}{l}55 \\
54 \% \\
47 \\
46 \%\end{array}$ & $\begin{array}{l}33 \\
32 \% \\
69 \\
68 \%\end{array}$ & $\begin{array}{l}38 \\
37 \% \\
64 \\
63 \%\end{array}$ & $\begin{array}{l}69 \\
68 \% \\
33 \\
32 \%\end{array}$ & $\begin{array}{l}33 \\
32 \% \\
69 \\
68 \%\end{array}$ & $\begin{array}{l}74 \\
73 \% \\
28 \\
27 \%\end{array}$ & $\begin{array}{l}07 \\
7 \% \\
95 \\
93 \%\end{array}$ & $\begin{array}{l}80 \\
78 \% \\
22 \\
22 \%\end{array}$ & $\begin{array}{l}71 \\
70 \% \\
31 \\
30 \%\end{array}$ & $\begin{array}{l}32 \\
31 \% \\
70 \\
69 \%\end{array}$ & - & - & - & - & - & - & - & - & - \\
\hline $\begin{array}{l}\text { Pseudo } \\
\text { Monas spp. } \\
(\mathrm{n}=55)\end{array}$ & $\mathrm{R}$ & - & - & - & - & - & - & - & $\begin{array}{l}31 \\
56 \% \\
24 \\
44 \%\end{array}$ & $\begin{array}{l}28 \\
51 \% \\
27 \\
49 \%\end{array}$ & $\begin{array}{l}12 \\
22 \% \\
43 \\
78 \%\end{array}$ & $\begin{array}{l}13 \\
24 \% \\
42 \\
76 \%\end{array}$ & $\begin{array}{l}41 \\
75 \%\end{array}$ & $\begin{array}{l}10 \\
18 \% \\
45 \\
82 \%\end{array}$ & $\begin{array}{l}16 \\
30 \% \\
39 \\
70 \%\end{array}$ & $\begin{array}{l}14 \\
25 \% \\
41 \\
75 \%\end{array}$ & $\begin{array}{l}43 \\
78 \%\end{array}$ & $\begin{array}{l}23 \\
42 \% \\
32 \\
58 \%\end{array}$ & $\begin{array}{l}24 \\
44 \% \\
31 \\
56 \%\end{array}$ & $\begin{array}{l}53 \\
96 \% \\
03 \\
04 \%\end{array}$ \\
\hline $\begin{array}{l}\text { E.Coli } \\
(\mathrm{n}=53)\end{array}$ & $\mathrm{R}$ & - & - & - & - & - & - & - & $\begin{array}{l}43 \\
81 \% \\
10 \\
19 \%\end{array}$ & $\begin{array}{l}22 \\
42 \% \\
31 \\
58 \%\end{array}$ & $\begin{array}{l}08 \\
15 \% \\
45 \\
85 \%\end{array}$ & $\begin{array}{l}06 \\
11 \% \\
47 \\
89 \%\end{array}$ & $\begin{array}{l}12 \\
23 \% \\
41 \\
77 \%\end{array}$ & $\begin{array}{l}06 \\
11 \% \\
47 \\
89 \%\end{array}$ & $\begin{array}{l}11 \\
21 \% \\
42 \\
79 \%\end{array}$ & $\begin{array}{l}13 \\
25 \% \\
40 \\
75 \%\end{array}$ & $\begin{array}{l}07 \\
13 \% \\
46 \\
87 \%\end{array}$ & $\begin{array}{l}09 \\
17 \% \\
44 \\
83 \%\end{array}$ & $\begin{array}{l}20 \\
38 \% \\
33 \\
62 \%\end{array}$ & - \\
\hline 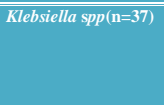 & $\mathrm{R}$ & - & - & - & - & - & - & - & $\begin{array}{l}21 \\
57 \% \\
16 \\
43 \%\end{array}$ & $\begin{array}{l}18 \\
49 \% \\
19 \\
51 \%\end{array}$ & $\begin{array}{l}06 \\
16 \% \\
31 \\
84 \%\end{array}$ & $\begin{array}{l}\text { Nil } \\
37 \\
100 \%\end{array}$ & $\begin{array}{l}04 \\
11 \% \\
33 \\
89 \%\end{array}$ & $\begin{array}{l}\text { Nil } \\
37 \\
100 \%\end{array}$ & $\begin{array}{l}05 \\
14 \% \\
32 \\
86 \%\end{array}$ & $\begin{array}{l}03 \\
08 \% \\
34 \\
92 \%\end{array}$ & $\begin{array}{l}\text { Nil } \\
37 \\
100 \%\end{array}$ & $\begin{array}{l}09 \\
24 \% \\
28 \\
72 \%\end{array}$ & $\begin{array}{l}08 \\
22 \% \\
29 \\
71 \%\end{array}$ & - \\
\hline Proteus spp $(\mathrm{n}=11)$ & $\mathrm{R}$ & - & - & - & - & - & - & - & $\begin{array}{l}10 \\
91 \% \\
01 \\
09 \%\end{array}$ & $\begin{array}{l}09 \\
82 \% \\
02 \\
18 \%\end{array}$ & $\begin{array}{l}04 \\
36 \% \\
07 \\
64 \%\end{array}$ & $\begin{array}{l}04 \\
36 \% \\
07 \\
64 \%\end{array}$ & $\begin{array}{l}06 \\
55 \% \\
05 \\
45 \%\end{array}$ & $\begin{array}{l}02 \\
18 \% \\
09 \\
82 \%\end{array}$ & $\begin{array}{l}03 \\
27 \% \\
08 \\
73 \%\end{array}$ & $\begin{array}{l}07 \\
64 \% \\
04 \\
36 \%\end{array}$ & $\begin{array}{l}04 \\
36 \% \\
07 \\
64 \%\end{array}$ & $\begin{array}{l}06 \\
55 \% \\
05 \\
45 \%\end{array}$ & $\begin{array}{l}09 \\
82 \% \\
02 \\
18 \%\end{array}$ & - \\
\hline $\begin{array}{l}\text { Strepto } \\
\text { Coccus spp } \\
(\mathrm{n}=8)\end{array}$ & $\mathrm{s}$ & $\begin{array}{l}03 \\
38 \% \\
05 \\
62 \%\end{array}$ & $\begin{array}{l}01 \\
13 \% \\
07 \\
87 \%\end{array}$ & $\begin{array}{l}03 \\
38 \\
\\
05 \\
62 \%\end{array}$ & $\begin{array}{l}05 \\
63 \% \\
03 \\
37 \%\end{array}$ & $\begin{array}{l}03 \\
38 \% \\
05 \\
62 \%\end{array}$ & $\begin{array}{l}04 \\
50 \% \\
04 \\
50 \%\end{array}$ & $\begin{array}{l}02 \\
25 \% \\
06 \\
75 \%\end{array}$ & $\begin{array}{l}06 \\
75 \% \\
\\
02 \\
25 \%\end{array}$ & $\begin{array}{l}05 \\
63 \% \\
03 \\
37 \%\end{array}$ & $\begin{array}{l}02 \\
25 \% \\
06 \\
75 \%\end{array}$ & - & - & - & - & - & - & - & - & - \\
\hline $\begin{array}{l}\text { Citro } \\
\text { Bacter } s p p \\
(\mathrm{n}=2)\end{array}$ & $\mathrm{R}$ & - & - & - & - & - & - & - & $\begin{array}{l}01 \\
50 \% \\
01 \\
50 \%\end{array}$ & $\begin{array}{l}01 \\
50 \% \\
01 \\
50 \%\end{array}$ & $\begin{array}{l}\text { Nil } \\
\\
02 \\
100 \\
\%\end{array}$ & $\begin{array}{l}\text { Nil } \\
02 \\
100 \%\end{array}$ & $\begin{array}{l}\text { Nil } \\
02 \\
100 \%\end{array}$ & $\begin{array}{l}\text { Nil } \\
02 \\
100 \%\end{array}$ & $\begin{array}{l}\text { Nil } \\
\\
02 \\
100 \\
\%\end{array}$ & $\begin{array}{l}\text { Nil } \\
\\
02 \\
100 \\
\%\end{array}$ & $\begin{array}{l}\text { Nil } \\
\\
02 \\
100 \%\end{array}$ & $\begin{array}{l}01 \\
50 \% \\
01 \\
50 \%\end{array}$ & $\begin{array}{l}01 \\
50 \% \\
01 \\
50 \%\end{array}$ & $\begin{array}{l}- \\
-\end{array}$ \\
\hline $\begin{array}{l}\text { Entero } \\
\text { Bacter } s p p \\
(\mathrm{n}=2)\end{array}$ & $\mathrm{R}$ & - & - & - & - & - & - & - & $\begin{array}{l}02 \\
100 \% \\
\text { Nil }\end{array}$ & $\begin{array}{l}02 \\
100 \\
\% \\
\text { Nil }\end{array}$ & $\begin{array}{l}01 \\
50 \% \\
01 \\
50 \%\end{array}$ & $\begin{array}{l}\text { Nil } \\
02 \\
0200 \%\end{array}$ & $\begin{array}{l}\text { Nil } \\
02 \\
100 \%\end{array}$ & $\begin{array}{l}\text { Nil } \\
02 \\
100 \%\end{array}$ & $\begin{array}{l}01 \\
50 \% \\
01 \\
50 \%\end{array}$ & $\begin{array}{l}01 \\
50 \% \\
\\
01 \\
50 \%\end{array}$ & $\begin{array}{l}\text { Nil } \\
02 \\
02 \%\end{array}$ & $\begin{array}{l}02 \\
100 \% \\
\text { Nil }\end{array}$ & $\begin{array}{l}02 \\
100 \% \\
\text { Nil }\end{array}$ & - \\
\hline $\begin{array}{l}\begin{array}{l}\text { Enterococci spp } \\
(\mathrm{n}=0 \mathrm{ne})\end{array} \\
\end{array}$ & S & $\begin{array}{l}01 \\
100 \\
\% \\
\text { Nil }\end{array}$ & $\begin{array}{l}\text { Nil } \\
\\
01 \\
100 \\
\%\end{array}$ & $\begin{array}{l}\text { Nil } \\
\\
01 \\
100 \\
\%\end{array}$ & $\begin{array}{l}01 \\
100 \\
\% \\
\text { Nil }\end{array}$ & $\begin{array}{l}\text { Nil } \\
\\
01 \\
100 \\
\%\end{array}$ & $\begin{array}{l}01 \\
100 \\
\% \\
\text { Nil }\end{array}$ & $\begin{array}{l}\text { Nil } \\
\\
01 \\
100 \\
\%\end{array}$ & $\begin{array}{l}01 \\
100 \% \\
\text { Nil }\end{array}$ & $\begin{array}{l}01 \\
100 \\
\% \\
\mathrm{Nil}\end{array}$ & $\begin{array}{l}\text { Nil } \\
\\
01 \\
100 \\
\%\end{array}$ & - & - & - & - & - & - & - & - & - \\
\hline
\end{tabular}

(S-Sensitivity, R-Resistanat, n-number of isolates, CD-Clindamycin, CX-Cefoxitin, CIP-Ciprofloxacin, DODoxycyclin, E-Erethromycin, LZ-Lenezolid, P-Penicillin, AK-Amikacin, LE-Levofloxacin, COT-Cotrimaxazole, AMC-Amoxycyclin=Clavulonic acid, CAZ-Caftazidime, CFM-Cefezime, CPZ-Cefoperazone, CXM-Cefuroxime, OF-Ofloxacin, PIT-Piperacilline + Tazobactum, IPM-Imipenem) 
Table.7 Isolation of MRSA and ESBL producer in wound infections

\begin{tabular}{|l|l|l|l|}
\hline Organisms isolated & Total no. of isolates & MRAS (\%) & $\begin{array}{l}\text { ESBL Produceres } \\
(\%)\end{array}$ \\
\hline S.aureus & 102 & $69(67.65 \%)$ & - \\
\hline $\begin{array}{l}\text { Gram negative } \\
\text { organisms }\end{array}$ & 160 & - & $61(38.12 \%)$ \\
\hline
\end{tabular}

Infection in a wound delays healing, causes wound breakdown, dehiscence, prolongation hospital stay, increased trauma and treatment cost. (Akinjogunla et al., 2009) Wound infection is an importatant cause of morbidity and mortality among surgical patients.

Infections of hospital acquired wounds are among the leading nosocomial causes of morbidity and increasing medical expenses. (Giacometti et al., 2000) The control of wound infections has become more challenging due to wide spread bacterial resistance to antibiotics and to a greater incidence of infections caused by Methicillin resistance S.aureus (MRSA) and polymicrobic flora. (Akinjogunla et al., 2009)

The knowledge of the causative agents of wound infection has therefore proved to be helpful in the selection of emphiric antimicrobial therapy and on infection control measures in hospital.

The present study was carried out from January 2012 to December 2012. Total 303 wound swabs or pus samples were received out of which $202(66.66 \%)$ were culture positive whereas in 101 (33.33\%) of cases there was no growth. In the present study culture positivity of wound infection was $66.66 \%$ which was in accordance with Azane and Beyene (2011). -70.5\%, Egbe et al., (2011)-64.8\%, Karia et al., (2013)-54.09\%, Anubumani et al., (2006)-47\% Whereas Sisirak et al., (2010), Shitto et al., (2002), and Mordi et al., (2009) reported high rate of culture positivity (84\%), (95\%) and (97.5\%) respectively.

In the present study out of 271 isolates, 160 $(59.04 \%)$ isolates were Gram negative and $111(40.96 \%)$ isolates were Gram positive. This was in accordance with Azene et al., (2011), where Gram negative isolates were $56.6 \%$ and Gram positive isolates were 43.4\%, Zafer et al., (2007), Goswami et al., (2011) and Mohanty et al., (2004) have also reported higher isolation of Gram negative organisms in a range of $50-70 \%$ as compare to Gram positive organisms in a range of $30-$ $49 \%$ in their study.

The most common organism isolated from wound infections in this study was S.aureus 102 (37.63\%) followed by Pseudomonas spp 55(20.30\%), E.Coli 53(19.56\%), Klebsiella spp 37(13.65\%), Proteus spp 11(4.05\%), Strptococcus spp 8(2.95\%), Citrobacter spp and Enterobacter spp 2(0.74\%; each and Enterococci spp one $(0.73 \%)$. The proportion of bacterial isolates was in agreement with previous study done by Azene et al., (2011) ${ }^{17}$, where S.aureus (41.6\%) was the most common isolate in their study followed by Pseudomonas spp (18.4\%), E.Coli (16.4\%), Proteus spp (11.0\%), Citrobacter and Enterobacter spp (4.2\%) each, and Klebsiella spp, (2.4\%). Several previous studies (Zafer $e t$ al., 2007, Anubamani et al., 2006, Mordi et al., 2009, Karia et al., 2013 and Egbe et al., 2011) reported S.aureus was the most common organism followed by Pseudomonas spp, E.Coli, Klebsiella spp, Proteus spp, and Streptococcus spp. Whereas Sule et al., 
(2002) reported Klebsiella spp was the most prevalent in the obsteritcs and gynaecological wounds and P.aeruginosa was the commonest in orthopaedics wounds. This might be due to geographical variations because bacterial etiology can show geographic variations and may even vary over time within a population.

The culture positivity of pathological wound infection was $67.69 \%$, trauma wound infection was $57.14 \%$, and post-operative wound infection was $67.33 \%$ in the present study. In the previous study done by Mordi et al., (2009) and Pramodhini et al., (2012) reported $100 \%$ culture positivity in pathological wound infection in their study.

Mordi et al., (2009) and Akinjogunla et al., (2009) reported $97.85 \%$ and $97.5 \%$ culture positivity in trauma wound infection respectively.

Shanthi et al., (2012) reported 67\% incidence of post-operative wound infection which is similar to our study. Whereas in several previous studies done by Ranjan et al., (2010), Kownher et al., (2008) and Patel et al., (2012) reported higher incidence of postoperative wound infection i.e. $91 \%$, $88.46 \%$, and $87.5 \%$ respectively. Above findings shows that incidence of all 3 types of wound infections is low in our hospital may be due to proper wound care, proper hygiene standard within a hospital. Postoperative sepsis rate in any hospital depends much on the case material, hospital environment, irrational use and availability of antibiotics.

In the present study isolated S.aureus were $78 \%$ sensitive to Amikacin, $73 \%$ to Linezolid, $70 \%$ to levofloxacin and $68 \%$ sensitive to Doxycyclin. In isolated Gram negative bacteria, Pseudomonas spp were $96 \%$ sensitive to imipenem and $56 \%$ sensitive to Leofloxacin. E.Coli, Klebsiella spp and Proteus spp were $81 \%, 57 \%$ and $91 \%$ sensitive to Amikacin respectively. Whereas Proteus spp were also $82 \%$ sensitive to Levofloxacin and Piperacillin-tazobactum, Klebsiella spp were $100 \%$ resistance to Amoxicillin-clavulanic acid, Cefexime and Cefuroxime. In the present study isolated most of the strains of Gram positive (S.aureus, Streptococcus spp and Enterococci spp) and Gram negative (E.Coli, Proteus sppand Enterobacter spp) organism were sensitive to Amikacin.

In previous studies reported that S.aureus was sensitive to Amikacin (Garg et al., 2009, Siguan et al., 1990, and Tayfour et al., 2005). Kaur et al., (2008) also reported that S.aureus was $74.4 \%$ sensitive to Amikacin and $97.5 \%$ sensitive to Linezolid. Whereas in the same study E.Coli, Klebsiella spp, Proteus spp, and P.aeruginosa were $80 \%, 60 \%, 50 \%$, and $50 \%$ sensitive to Amikacin respectively which were in accordance with present study. In previous study Goswami et al., (2011) reported that levofloxacin is sensitive to both Gram positive and Gram negative organisms. Amikacin is sensitive to both Gram positive and Gram negative isolates was reported in studies done by Siguan et al., (1990) and Dhar et al., (2007). Amikacin is effective for all Gram negative isolates was reported in various previous studies Shanthi et al., (2012), Kauer et al., (2008), Kownhar et al., (2008), and Dhar et al., (2007). Pseudomonas spp were $96 \%$ sensitive to imipenem in the present study. This was comparable with Tayfour et al., (2005), Mehta et al., (2007), and Ranjan et al., (2010) who reported that Klebsiella spp were $100 \%$ resistant to Amoxicillin-clavulanic acid which was similar to this study.

In the present study there was no single common antibiotic to which all isolated Gram positive and Gram negative isolates were increasingly resistant to routinely used antibiotics. 
In the present study we found $69(67.65 \%)$ Methicillin resistant S.aureus. This was less than previous study done by Etok et al., (2012) who reported 100\% Methicillin resistance in S.aureus (MRSA) from surgical wound infection. Pramodhini et al., (2012) and Mohanty et al., (2004) who reported $32.7 \%$ and $38.58 \%$ MRSA respectively.

In present study out of 160 Gram negative bacteria isolated, 61(38.12\%) were ESBL producers. This is comparable to that reported previously $(32.6 \%)$ by Pramodhini et al., (2012). Higher rate of ESBL producers were reported by Mohanty et al., (2004)-66.75\% and Shanthi et al., (2012)-65.2\% in their study.

Finally this study concludes that contracting wound infection remains an ongoing problem. The main culprits for wound infection are Methicillin resistant S.aureus, ESBL producing Gram negative bacteria like Pseudomonas spp, E.Coli, Klebsiella spp, and Proteus spp. Although complete eradication of wound infections is not possible however we recommend that by taking preventive measures, adopting prompt clean surgical procedures, instituting sensitivity based antibiotic treatment and proper surgical care of wounds, the incidence of wound infection can be reduced. Therefore there is need of ongoing studies on wound infections for isolating different causative organisms, to know its sensitivity pattern for management of wound infections and minimize emergence of drugs resistant organisms.

\section{Acknowledgements}

Authors are thankful to Dean, M.G.M. Medical College and Hospital, Aurangabad for granting the permission for this work.

\section{References}

Akinjogunla OJ, Adegoke AA, Mboto CI, Chukwudebelu IC, Udokang IP.
Bacteriology of automobile accident wound infection. Int J Med Sci 2009; 1(2): 19-22.

Anumani N, Kalyan J, Mallika M. Epidemiology and microbiology of wound infections. Indian Journal for the Practicing Doctor 2006; 3(5): 1-5.

Azene MK, and Beyene BA. Bacteriology and antibioGram of pathogens from wound infections at Dessie laboratory, North East Ethopia. Tanza J Health Res 2011; 13(4): 1-10.

Bowler PG, and Duerden BI, Armstrong DG. Wound microbiology and associated approaches to wound management. Clin Microbiol Rev 2001; 14(2): 244-69.

Church D, Elsayed S, Reid O, Winstone B, Lindsay R. Burn wound infections. Clin Microbiol Review 2006; 19(2): 403-34.

Clinical and Laboratory Standards Institute. Performance standard for antimicrobial susceptibility testing: Twenty second informational supplement. CLSI Document M100-S22, 2012; 32(3): 1188.

Collier M. Understanding wound inflammation. Nursing times 2003; 99(25): 63-64.

Dhar S, Saraf R, Singh K, Raina B. Microbiological profile of chronic burn wound among patients admitted in burn unit. J Med Edu Res 2007; 9(4): 182-85.

Egbe CA, Omeregie R, Jgbarumah IO, Onemu S. Microbiology of wound infections and its associated risk factors among patients of a tertiary hospital in Benin city, Nigeria. J Res Health Sci 2011; 11(2): 109-13.

Etok CA, Edem EN, Ochang E. Aetiology and antimicrobial studies of surgical wound infections in University of Uyo Teaching Hospital (UUTH) Uyo, Akwa Ibom state, Nigeria. Open Scientific Reports 2012; 1(7): 1-5.

Garg A, Sujatha S, Garg J Acharya NS, Parija SC. Wound infections secondary to 
snake bite. Journal of infection in developing countries 2009; 3(3): 22123.

Giacometti a, Cirioni O, Schimizzi AM, Del Prete MS, Barchiesi F, D'Errico MM, Petrlli E, Scalise G. Epidemiology and Microbiology of surgical wound infections. J Clin Microbiol 2000; 38(2): 918-22.

Goswami NN, Trivedi HR, Goswami APP, Patel TK, Tripathi CB. Antibiotic sensitivity profile of bacterial pathogens in postoperative wound infections at a tertiary care hospital in Gujrat, India. J Pharmcol Pharmcotherapeutic 2011; 2(3): 158-64.

Gottarup F, Melling A, Hollander DA. An overview of surgical site infections: aetiology, incidence and risk factors. Eur Wound Mgt Asso J 2005; 5(2): 1115.

Karia JB, Gadekar HB, Lakhani SJ. Study of bacterial profile of pus culture in Dhiraj General Hospital. Indian J Applied Basic Med Sci 2013; 15(20): 70-76.

Kaur J, Gulati VL, Aggarwal a, Gupta V. Bacteriological profile of osteomyelitis with special reference to Staphylococcus aureus. Indian J Pract Doctor 2008; 4(6): 1-7.

Kirby UMM, Bauer AW, Sherris TC, Truck M.antibiotic susceptibility testing by standardized single disc methods. Am J Clin Pathol 1966; 45: 493-96.

Kownhar H, Shankar EM, Vignesh R, Sekar $\mathrm{R}$, Velu V, Rao UA. High isolation rate of Staphylococcous aureus from surgical site infections in an Indian hospital 2008; 61(3): 758-60.

Mackie and McCartney. Practical Medical Microbiology. $13^{\text {th }}$ edi. Edr Colle JG, Duguid JP, Fraser AG, Marmion BP. Churchill Livingston, UK 1989.

Mehta M, Dutta P, Gupta V. Bacterial isolates from burn wound infections and their
antibiGram: a eight year study. Indian $\mathbf{J}$ Plast surg 2007; 40(1): 25-28.

Mohanty S, Kapil A, Dhawan B, Das BK. Bacteriological and antimicrobial susceptibility profile of soft tissue infections from North India. Indian J Med Sci 2004; 58(1): 10-15.

Mordi RM, and Momoh MI. Incidance of Proteus spp in wound infections and their sensitivity pattern in the university of Benin Teaching Hospital. Afr J Biotech 2009; 8(5): 725-30.

Njoku-Obi AN, and Ojiegbe GC. Resistance patterns of bacterial isolates from wound infections in a university teaching hospital. West Afr J Med 1989; 8(1): 29-34.

Patel SM, Patel MH, Patel SD, Soni ST, Kinariwala, Dipa M. surgical site infections: incidence and risk factors in a tertiary care hospital, Western India. National J Comm Med 2012; 3(2): 19396.

Pramodhini S, Tenmozhivalli PR, Selvi R, Dillirani V, Vasumathi A, Agatha D. Bacteriological profile of superficial and deep seated abscess and their antibiGram in a tertiary care hospital, South India. Indian J Med Specialities 2012; 3(2): 123-29.

Ranjan KP, Ranjan N, Bansal SK, Arora DR. Prevalence of Pseudomonas aeruginosa in postoperative wound infection in a Referral Hospital in Haryana India. J Lab Phy 2010; 2(2): 74-77.

Sani RA, Garba SA, Oyewole OA, Ibrahim A. Antibiotic resistance profile of Gram positive bacteria isolated from wound infections in Minna, Bida, Kontagora and suleja area of Niger State. J Health Sci 2012; 2(3): 19-22.

Shanthi J, Shankar T, Balagurunathan R. The scenario of postoperative wound infection from a Government hospital in South India. J Microbiol Biotechnol 2012; 2(3): 396-401. 
Shittu AO, Kolawole DO, Oyedepo EAR. A study of wound infections in two health institutions in ILE-IFE, Nigeria. Afr J Biomed Res 2002; 5: 97-102.

Siguan ss, Ang BS, Pala IM, Bacling RM. Aerobic surgical infection: A surveillance on microbiological etiology and antimicrobial sensitivity pattern of commonly used antibiotic. Philippino J Microbial infectious Disc 1990; 19(1): 27-33.

Sisirak M, Zuizdic A, Hukic M. MethicillinResistant Staphylococcous aureus (MRSA) as a cause of nosocomial wound infections. Bosnian J Basic Med Sci 2010; 10(1): 32-37.

Sule AM, Thanni Loa, sule Odu OA, Olusanya O. Bacterial pathogens associated with infected wounds in Ogun state University Teaching Hospital, Saguma, Nigeria. Afr J Clin expt Microbiol 2002; 3(1): 13-16.

Taiwo SS, Okesina AB, Onile BA. In vitro antimicrobial susceptibility pattern of bacterial isolates from wound infections in university of $\mathrm{O}$ Ilorin Teaching Hospital. Afr J Clin Expt Micribiol 2002; 3(1): 6-10.

Tayfour MA, Al-Ghamdi SM, Al-Ghamdi As. Surgical wound infections in King Fahad Hospital at Al-Baha, Saudi Med J 2005; 26(8): 1305-7.

Zafar A, Anwar N, Ejaz H. Bacteriology of infected wounds- A study conducted at children hospital, Lahore, J Biomed-8A 2007; 23: 1-4.

\section{How to cite this article:}

Bhalchandra M. H., S. D. Naik and Pramod Kumar Verma. 2018. Aerobic Bacterial Profile of Wound Infections and Its Sensitivity Pattern at Tertiary Care Hospital. Int.J.Curr.Microbiol.App.Sci. 7(06): 1668-1679. doi: https://doi.org/10.20546/ijcmas.2018.706.198 